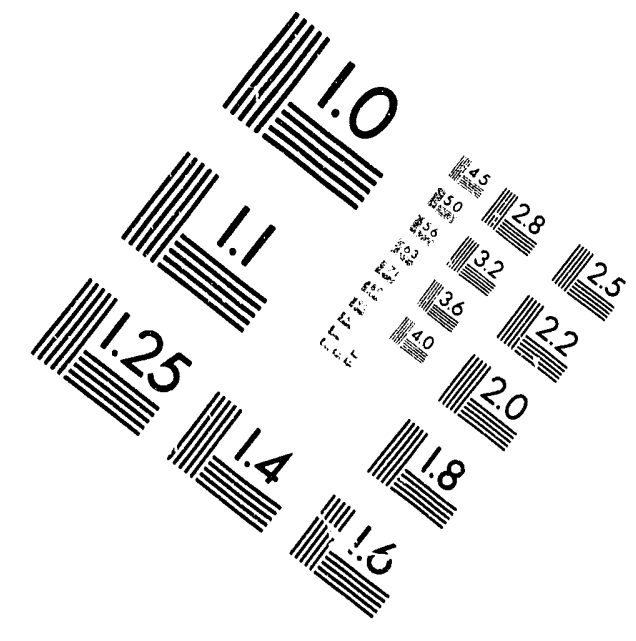

\title{
Centimeter
}

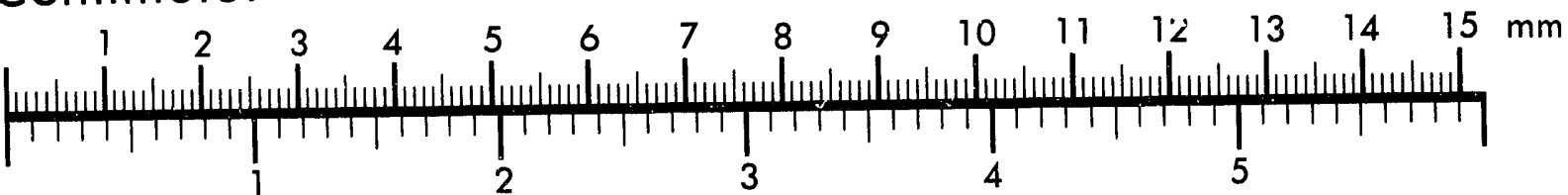

Inches
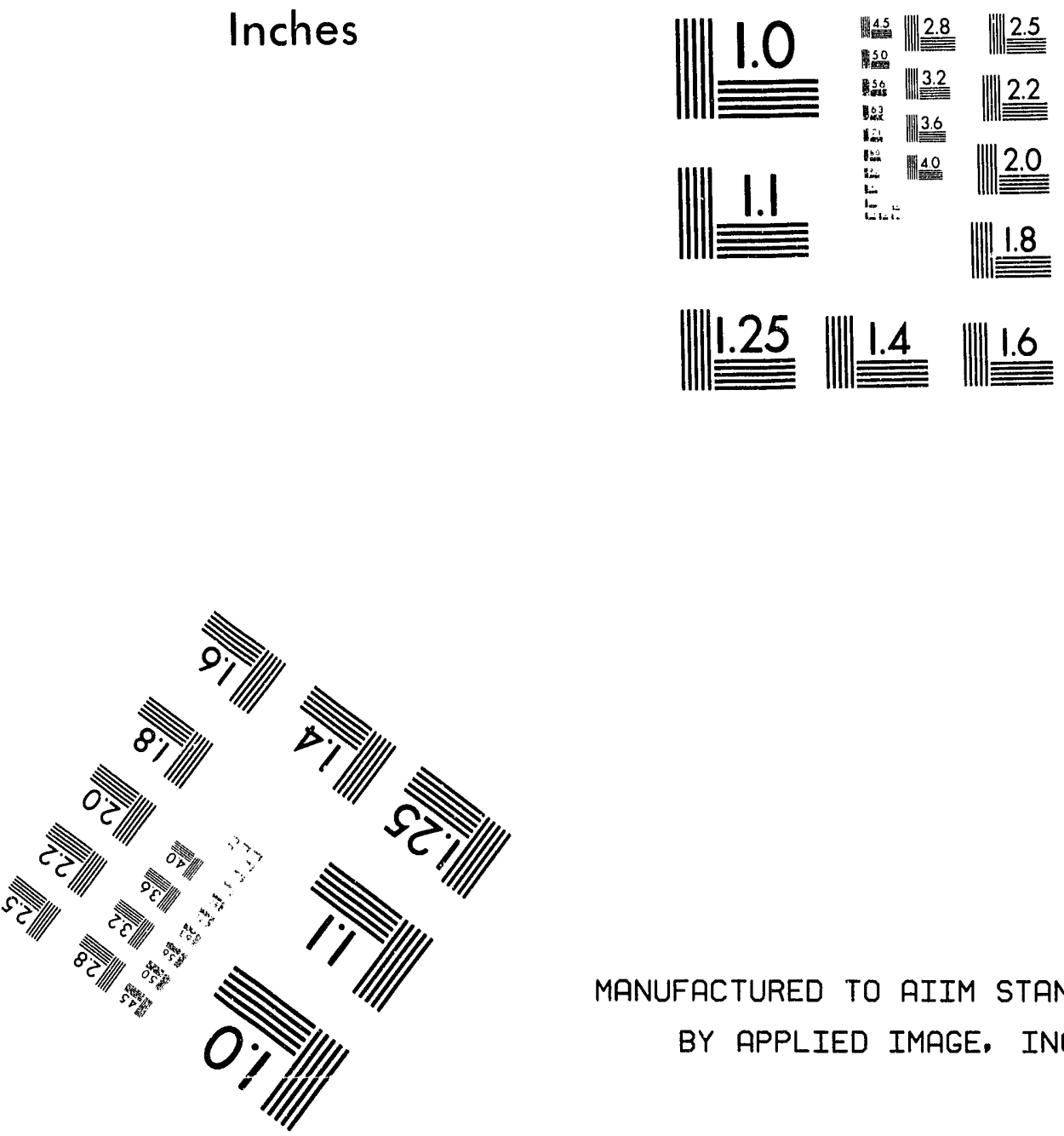

MANUFACTURED TO AIIM STANDARDS

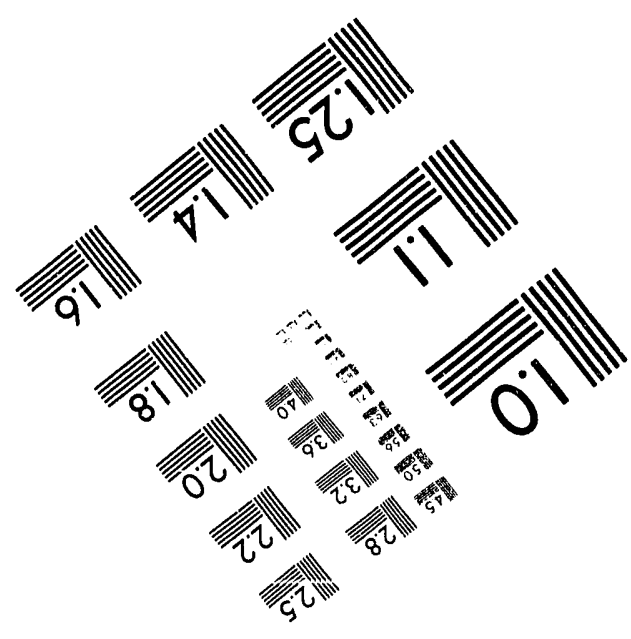



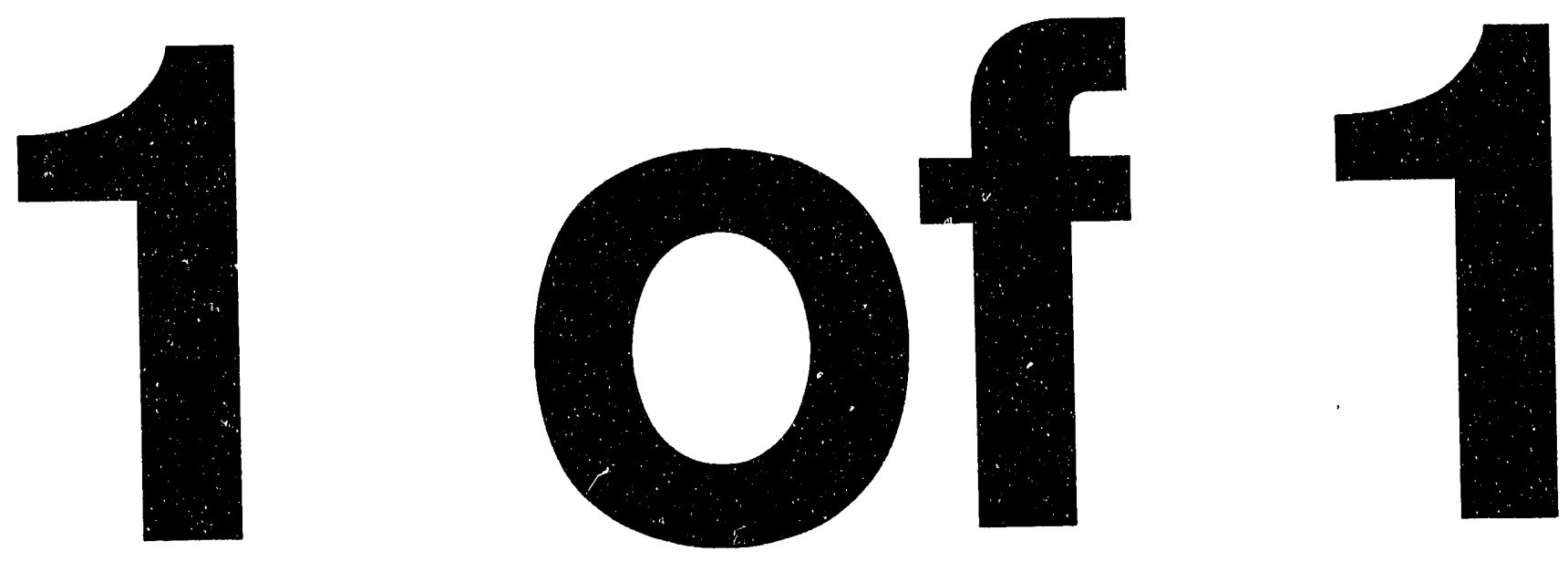


\title{
DIMENSIONALLY SIMILAR DISCHARGES WITH CENTRAL RF HEATING ON THE DIII-D TOKAMAK
}

\author{
by \\ C.C. PETTY, T.C. LUCE, and R.I. PINSKER
}

This is a preprint of a paper presented at the Tenth Topical Conference on Radio Frequency Power in Plasmas, April 1-3, 1993, Boston, Massachusetts, and to be printed in the Proceedings.

Work supported by

U.S. Department of Energy

Contract DE-AC03-89ER51114

GENERAL ATOMICS PROJECT 3466

APRIL 1993

\section{GENERAL ATOMICS

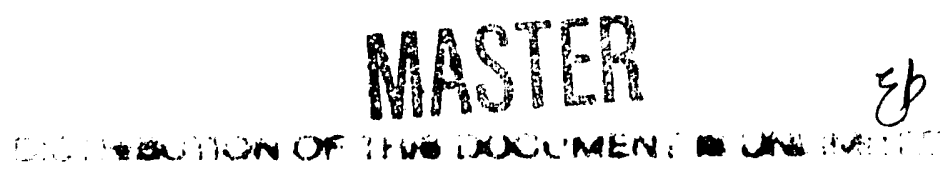




\title{
DIMENSIONALLY SIMILAR DISCHARGES WITH CENTRAL RF HEATING ON THE DII-D TOKAMAK
}

\author{
C.C Petty, T. C. Luce, and R.I. Pinsker \\ General Atomics, San Diego, CA 92186-9784
}

\begin{abstract}
The scaling of L-mode heat transport with normalized gyroradius is investigated on the DIII-D tokamak using central rf heating. A toroidal field scan of dimensionally similar discharges with central ECH and/or fast wave heating show gyro-Bohm-like scaling both globally and locally. The main difference between these results and those using NBI heating on DIII-D is that with rf heating the deposition profile is not very sensitive to the plasma density. Therefore central heating can be utilized for both the low- $B$ and high- $B$ discharges, whereas for NBI the power deposition is decidedly off-axis for the high- $B$ discharge (i.e., high density).
\end{abstract}

\section{INTRODUCTION}

The diffusion of heat across magnetic field lines is of fundamental importance to tokamaks. Diffusion processes can be categorized as being either short wavelength or long wavelength. For "dimensionally similar" discharges, defined as having identical values for all dimensionless parameters except the relative gyroradius (i.e., having $\nu^{*}, \beta$, $q, T_{\mathrm{e}} / T_{\mathrm{i}}, R / a, b / a$, etc., the same but not $\left.\rho^{*}\right)$, the diffusivity has a very simple scaling: $\chi \propto B^{-1} a^{-1 / 2}$ for microturbulence or gyro-Bohm-like scaling, or $\chi \propto B^{-1 / 3} a^{1 / 3}$ for macroturbulence or Bohm-like scaling. ${ }^{1}$ Nearly all theories for diffusion are gyroBohm-like; however, along a dimensionally similar path the empirical L-mode tokamak scalings (such as Goldston) have $\tau \propto B^{0}$ which is slightly worse than Bohm-like.

Comparing dimensionally similar discharges of the same size but different magnetic field should allow one to distinguish between a gyro-Bohm-like or a Bohm-like diffusion process. If the plasma transport is purely diffusive, then the global confinement should scale as $\tau \propto B a^{5 / 2}$ for gyro-Bohm-like diffusion or $\tau \propto B^{1 / 3} a^{5 / 3}$ for Bohm-like diffusion. A technical difficulty with this experiment is keeping the auxiliary heating profile similar for the low- $B$ and high- $B$ discharges, especially with NBI heating. Central heating with rf power is ideally suited for this type of experiment since the deposition profile is relatively independent of the plasma density.

\section{EXPERIMENTAL RESULTS}

For DIII-D, two forms of central rf heating are available to directly heat electrons: $60 \mathrm{GHz} \mathrm{ECH}$ and $60 \mathrm{MHz}$ fast wave (FW). The $\mathrm{ECH}$ is resonant centrally at $2 \mathrm{~T}$ for fundamental absorption and $1 \mathrm{~T}$ for second harmonic absorption. The $\mathrm{FW}$ in the direct electron heating regime is always absorbed near the plasma center, regardless of the magnetic field, since the damping increases with electron temperature. ${ }^{2}$

The fast wave current drive (FWCD) database was examined for pairs of shots which were dimensionally similar. Table I shows some dimensionless parameters for the best matched pair of $\mathrm{xf}$ discharges. These divertor plasmas are in $\mathrm{L}$-mode and are 
Table I

Dimensionless parameters for dimensionally similar discharges with central rf heating

\begin{tabular}{lll}
\hline \hline & $B_{\mathrm{T}}=1.07 \mathrm{~T}$ & $B_{\mathrm{T}}=1.99 \mathrm{~T}$ \\
\hline$R / a$ & 2.7 & 2.7 \\
$b / a$ & 1.7 & 1.8 \\
$q_{95}$ & 6.9 & 6.7 \\
$\ell_{\mathrm{i}}$ & 1.8 & 1.8 \\
$\beta_{\mathrm{p}}$ & 0.37 & 0.34 \\
$Z_{\text {eff }}$ & 3.2 & 3.3 \\
\hline \hline
\end{tabular}

fueled by deuterium gas puffing. The high- $B$ shot had co-ECCD and co-FWCD while the low- $B$ shot had co-FWCD only. The ratio of $\mathrm{rf}$-to-ohmic power was nearly the same (5:1) for these two plasmas. More than $70 \%$ of the total heating power was deposited inside $\rho=1 / 3$ for both discharges.

Figure 1 shows the profiles of $\beta, \nu^{*}, T_{\mathrm{e}} / T_{\mathrm{i}}$, and the absorbed power fraction for the two rf-heated dimensionally similar discharges. Ion temperature profiles were obtained by pulsing the neutral beams for approximately $10 \mathrm{msec}$ to obtain CER data. The profiles are well matched throughout the plasma. Although not shown, the density and temperature profile scale lengths are also well matched for these two discharges.

Table II shows the heating power, stored energy, and global confinement for these two discharges. The confinement time scaled like $\tau \propto B^{1.2}$, which is slightly stronger than gyro-Bohm-like. Since the global confinement exhibits gyro-Bohm-like scaling, the local transport should also be gyro-Bohm-like. The local transport is determined from a power balance analysis code. The ECH and FW deposition profiles and current drive are calculated by ray tracing codes. For the $B_{\mathrm{T}}=1.99 \mathrm{~T}$ discharge, a large fraction of the FW power is absorbed centrally by second harmonic heating of the $\approx 2 \%$ hydrogen minority. This complicates a two-fluid analysis of the transport, since the partition of the FW power to ions and electrons is not accurately known. Therefore in order to eliminate this uncertainty, a one-fluid transport analysis is performed. The one-fluid heat diffusivity for each discharge was calculated using

$$
\chi_{\text {eff }}=-\frac{q_{\mathrm{e}}+q_{\mathrm{i}}}{n_{\mathrm{e}} \nabla T_{\mathrm{e}}+n_{\mathrm{i}} \nabla T_{\mathrm{i}}} .
$$

The ratio of the $\chi_{\text {eff }}$ in the $1.99 \mathrm{~T}$ discharge to that found in the $1.07 \mathrm{~T}$ discharge is shown in Fig. 2. Also shown are the ratios for Bohm-like and gyro-Bohm-like transport scaling. The experimental data for the rf centrally heated plasmas is again clearly much closer to gyro-Bohm-like than Bohm-like for all radii. The $\mathrm{rf}$ heating profiles were similar only outside $\rho=1 / 3$, which may explain the deviation from gyro-Bohmlike scaling inside that radius, although sawtooth transport may also be an important effect.

\section{DISCUSSION}

Dimensionally similar discharges with central of heating exhibit gyro-Bohm-like scaling both globally and locally. This is in contrast to previous results from DIII-D 

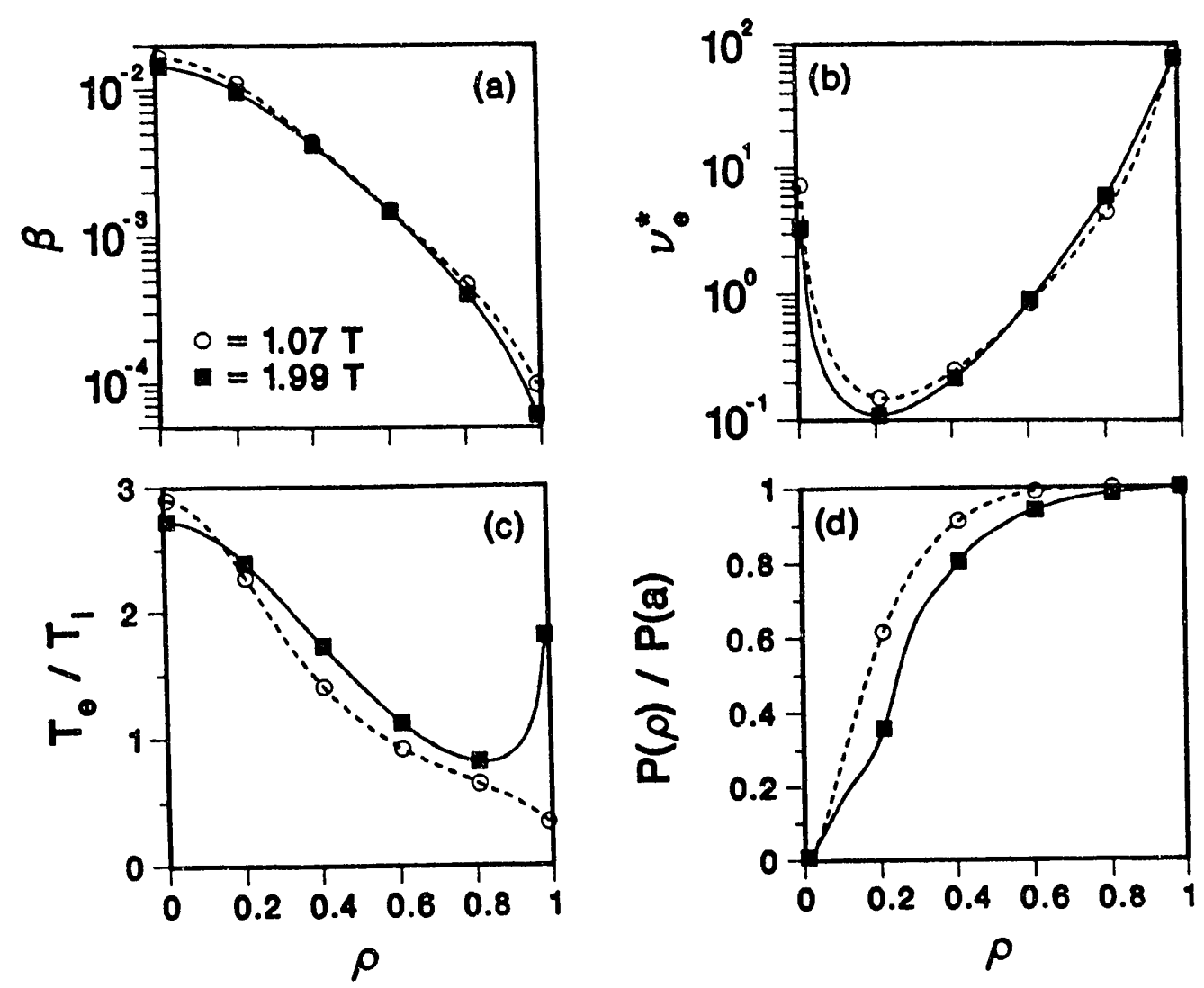

Fig. 1. Profiles of dimensionless parameters for two dimensionally similar discharges with central if heating.

Table II

Global confinement parameters for dimensionally similar discharges with central rf heating

\begin{tabular}{lll}
\hline & $B_{\mathrm{T}}=1.07 \mathrm{~T}$ & $B_{\mathrm{T}}=1.99 \mathrm{~T}$ \\
\hline$P_{\text {rf }}$ & $0.90 \mathrm{MW}$ & $1.45 \mathrm{MW}$ \\
$P_{\text {oh }}$ & $0.16 \mathrm{MW}$ & $0.29 \mathrm{MW}$ \\
$W$ & $39 \mathrm{~kJ}$ & $131 \mathrm{~kJ}$ \\
$\tau$ & $36 \mathrm{msec}$ & $75 \mathrm{msec}$ \\
\hline
\end{tabular}

with NBI heating where the global confinement time followed Bohm-like scaling or worse for a sirnilar L-mode $B$-scaling experiment. ${ }^{1}$ One difference between these two experiments is that with rf heating the deposition profile is not very sensitive to the plasma density. Therefore central rf heating can be utilized for both the low- $B$ and high- $B$ discharges, whereas for NBI the power deposition is decidedly off-axis for the high- $B$ discharge (i.e., high density). However, when dimensionally similar discharges 


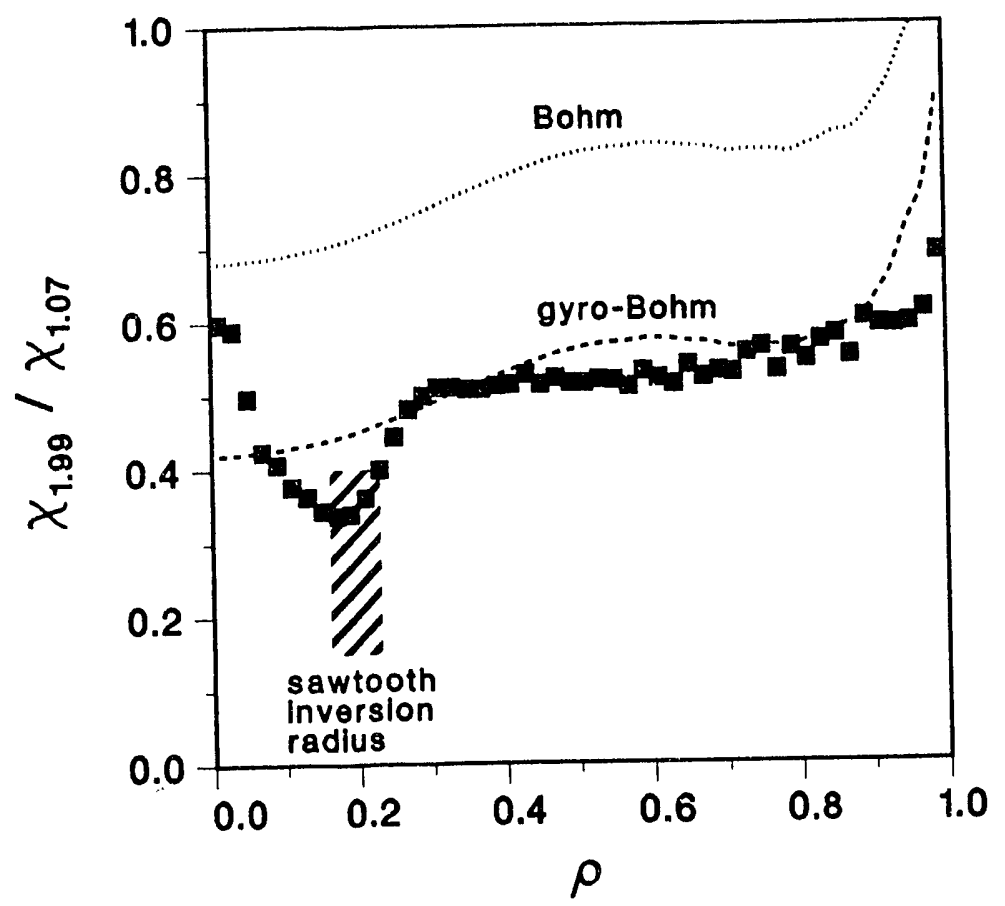

Fig. 2. Ratio of $1.99 \mathrm{~T}$ and $1.07 \mathrm{~T}$ one-fluid heat diffusivity versus normalized radius (solid squares).

with central ICRH were studied on JET, the transport still appeared to be Bohmlike. $^{3}$ The different experimental results between DIII-D and JET may be due to systematic differences in the operating regimes. For DIII-D the rf power directly heats the electrons resulting in $T_{\mathrm{e}} / T_{\mathrm{i}}$ well above unity, whereas $T_{\mathrm{e}} \approx T_{\mathrm{i}}$ in the JET experiments. In addition, the if experiments on DIII-D operated at higher safety factor and lower density, and thus higher $Z_{\text {eff }}$ than the JET experiments. Future experiments on DIII-D at higher rf power will address these systematic differences.

\section{ACKNOWLEDGMENT}

This is a report of work sponsored by the U.S. Department of Energy under Contract No. DE-AC03-89ER51114.

\section{REFERENRTS}

1. R.E. Waltz, J.C. DeBoo, and M.N. Rosenbluth, Phys. Rev. Lett. 65, 2390 (1990).

2. C.C. Petty, R.I. Pinsker, M.J. Mayberry, et al., Phys. Rev. Lett. 69, 289 (1992).

3. J.P. Christiansen, B. Balet, D. Boucher, et al., Plasma Phys. and Contr. Fusion 34, 1881 (1992). 

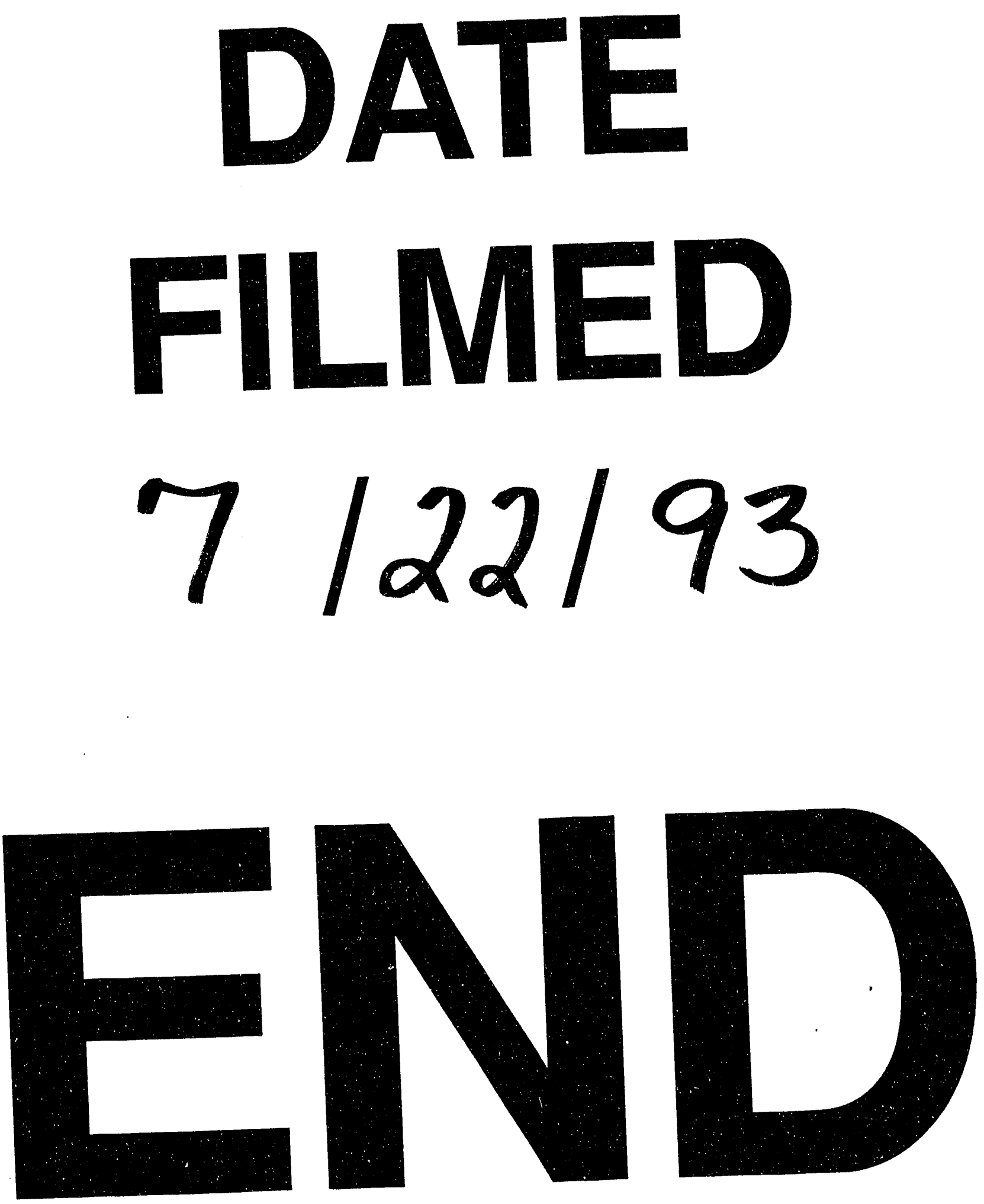
